\title{
Single-stage C6-7 ACDF with T1-2 Oblique Keyhole Transcorporeal Disectomy to Treat Cervico-thoracic Tandem Disc Herniation: A Case Report
}

\author{
Kang Suk Moon ${ }^{1}$, MinSeung Jeong ${ }^{2}$, Sang-Ho Lee ${ }^{3}$, Oon-Ki Baek ${ }^{1}$ \\ ${ }^{1}$ Department of Neurosurgery, Wooridul Spine Hospital, Gimpo Airport, Seoul, Korea \\ ${ }^{2}$ Department of Minimally Invasive and Endoscopic Spine Surgery, Espalda Saludable, Hospital Angeles, Ciudad de Mexico, Mexico \\ ${ }^{3}$ Department of Neurosurgery, Wooridul Spine Hospital, Seoul, Korea
}

Received: November 15, 2021

Accepted: January 2, 2022

Corresponding Author:

Kang Suk Moon, MD, PhD

Department of Neurosurgery,

Wooridul Spine Hospital, Gimpo

Airport, 70 Haneul-gil, Gangseo-gu,

Seoul 07505, Korea

Tel:+82-2-2660-7500

Fax: +82-2-2660-7599

E-mail: nsdrmoonks@gmail.com
Symptomatic cervico-thoracic tandem disc herniation occurs very rarely. On the other hand, cervical disc herniations are common and may be treated via a variety of surgical procedures. Symptomatic upper thoracic disc herniations are extremely rare, and use of a surgical approach in their treatment is controversial due to the narrow operative space within which surgical procedures must be performed. We report an extremely rare case of symptomatic tandem C6-7 and T1-2 disc herniation successfully treated via single-stage, single-incision, C6-7 anterior cervical decompression and fusion, and T1-2 oblique keyhole transcorporeal discectomy. This is the first symptomatic cervico-thoracic tandem disc herniation with its treatment.

Key Words: Disc herniation, Thoracic vertebrae, Cervical vertebrae

\section{INTRODUCTION}

Symptomatic cervico-thoracic tandem disc herniation occurs very rarely. Cervical disc herniation is a common pathology, which can be treated via several different surgical procedures [1]. Anterior cervical decompression and fusion (ACDF) remains the gold standard for treatment of symptomatic cervical disc herniation resistant to conservative treatment [1,2]. Symptomatic thoracic disc herniation (TDH) is very rare compared to disc herniation at cervical and lumbar regions, and occurs in 1 of 1,000,000 patients annually, comprising up to $4 \%$ of all surgical procedures $[3,4]$. Further, $75 \%$ of TDHs occur between the T8 and T12 levels [3].

The first case of T1-2 disc herniation was reported in 1954 by Sivien and Karavitis [4]. The optimal surgical approach for upper TDH treatment remains controversial [4]. Both anterior and posterior approaches have associated risks. When performing T1-2 surgery, use of the anterior approach is very challenging due to the narrow operative space available. Particularly, accessing the T1-2 level without manubriectomy, sternotomy or claviculectomy remains an issue [3,4]. On the other hand, use of the posterior approach in T1-2 surgery is associated with a high rate of neurological injury [5]. Here, we present an extremely rare case of symptomatic, tandem C6-7 and T1-2 disc herniation that was successfully treated using single-stage, single-incision C6-7 ACDF and T1-2 oblique keyhole transcorporeal discectomy.

\section{CASE REPORT}

\section{Initial Findings}

A 78-year-old-man without history of degenerative diseases

Copyright (C) 2022 Korean Minimally Invasive Spine Surgery Society

This is an Open Access article distributed under the terms of the Creative Commons Attribution Non-Commercial License (http://creativecommons.org/licenses/by-nc/4.0/) which permits unrestricted non-commercial use, distribution, and reproduction in any medium, provided the original work is properly cited. 
and previous surgeries presented with a 1-year history of cervico-thoracic back pain who recently developed progressive motor weakness of both hands and both legs. A neurological examination revealed that the patient experienced numbness in both hands, and hand intrinsic muscle weakness. Hyperactive brachioradialis deep tendon reflexes and positive Hoffmann's sign was observed. Paresthesia and weakness of the lower extremities with gait instability were also observed. Magnetic resonance imaging (MRI) showed a C6-7 and T1-2 disc extrusion with signs of spinal cord compression including cord signal change a levels corresponding to the extrusions (Figure 1).

\section{Operative Technique}

The patient was placed in a supine position under nerve integrity monitor (NIM). After localizing the C6-7 disc space with fluoroscope, a Smith-Robinson approach was used. A 5-cm transverse incision was made at the $\mathrm{C} 7$ level. The sternocleidomastoid (SCM) and the carotid artery were localized and moved laterally. Subsequently, the trachea and esophagus were localized and moved medially. Then, when the longus colli muscle, an anatomic landmark, was visible they were dissected unilaterally in the medial to lateral direction. The C6-7 vertebral body (VB) was localized, and its identity was verified using a fluoroscope. The C6-7 disc was localized, and complete discectomy was performed using a microscope.

After the C6-7 discectomy performed successfully, we removed the retractor and attempted to localize the T1 VB. Achieving a true lateral view of T1-2 was very difficult due to the shoulder anatomy of the patient. Therefore, oblique views to confirm the location of the T1 VB were needed. After con- firming the location of the $\mathrm{T} 1 \mathrm{VB}$, we identified the precise keyhole drill point that was located during preoperative planning (Figure 2). Using a fluoroscope and microscope for assistance, high-speed diamond burr drilling was performed in oblique, lateral to central and cephalad to caudal directions, making a keyhole that allowed us to precisely reach the central, T1-2 disc space. After confirming the position of the tip of the burr in the T1-2 disc space with fluoroscope, the discectomy was performed without any complications.

Once the decompression with discectomy at the T1-2 level was completed, we proceeded to place the cage and anterior plate was placed at the C6-7 level. Cage placement was performed at the end of the procedure since we wanted to check for bleeding at the C6-7 level. The correct position of the cage and plate was confirmed, each layer was sutured, and drainage

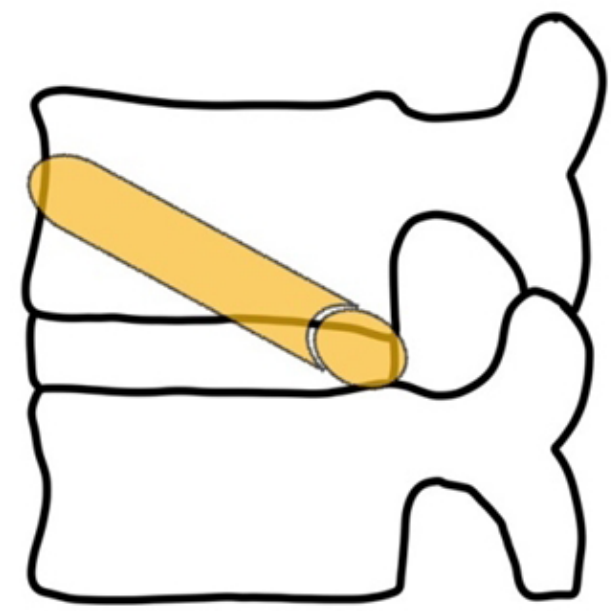

Figure 2. A schematic image of the oblique keyhole transcorporeal approach is shown.
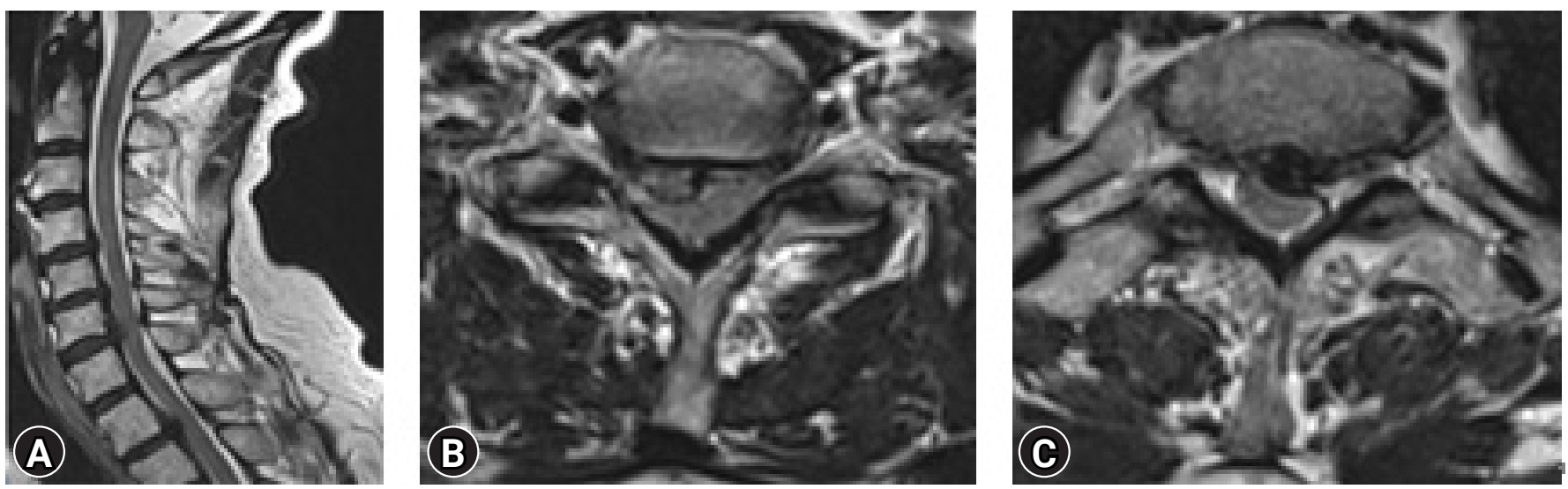

Figure 1. (A) Preoperative, sagittal T2 magnetic resonance imaging (MRI) of C6-7 and T1-2 disc herniation with signs of cord compression. (B) A preoperative, axial T2 MRI shows right disc herniation at C6-7. (C) A preoperative axial T2 MRI revealing central disc herniation at T1-2 is shown. 
was placed. The total duration of surgery was 2.5 hours.

\section{Postoperative Course}

Immediate cervico-thoracic back pain improvement was observed. Upper and lower extremity weakness, symptoms of myelopathy, as well as gait instability also improved significantly. The patient was discharged 14 days after surgery without any complications. Postoperative MRI scans showed complete removal of disc fragments from C6-7 and T1-2 regions (Figure $3)$. The diameter of keyhole entry at the vertebral body was approximately $5 \mathrm{~mm}$.

\section{DISCUSSION}

Symptomatic tandem disc herniation of the cervical and thoracic spine occurs only in extremely rare cases. Several cases of tandem disc herniation of the cervical and lumbar, but not thoracic spine have been reported. The reason for this is likely because intervertebral disc herniation rarely occurs at the T1-2 level. This area is generally obstructed by the shoulder of the patient when examined via X-ray, and is located at the distal end of computed tomography (CT) or MRI examination findings [6]. Surgical treatment for thoracic disc herniation is indicated in patients with symptoms of spinal cord compression that are confirmed via MRI, and for those with radicular symptoms for whom conservative management has failed [7].

Nonetheless, surgical treatment of thoracic disc herniation remains a challenge due to its anatomy [7]. The spinal cord at the thoracic level fills most of the spinal canal, making it vulnerable to disc and intraoperative traction injuries [7]. Until the 1990s, T1-2 herniation was treated via the posterior approach with laminectomy exclusively. However, these surgeries were characterized by very high death rates (up to $50 \%$ ) and an even higher morbidity levels of up to $70 \%$ [7]. Hence, use of the posterior approach for treating centrally located pathologies remains challenging. The presence of a narrow space makes it hard to retract the spinal cord without causing additional cord compression and cerebrospinal fluid leakage [8].

Anterior approaches were introduced as an alternative to laminectomy. Moreover, modern minimally invasive techniques were introduced such as thoracoscopic and endoscopic discectomy [7]. It remains obvious that the sternum, thoracic kyphosis, and clavicle restrict access to the pathology [8], and accessing the T1-2 disc space remains an issue, particularly with respect to whether it is possible for a surgeon to access the level without manubriectomy, sternotomy or claviculectomy [8]. Both posterior and anterior approaches are associated with high rates of complications.

Costotransversectomy and transthoracic approaches have high risk of pleural injury, hemothorax, subclavian vein injury and laryngeal nerve dysfunction, with complication rates of $54 \%$ and the potential for massive blood loss $(1,500-200 \mathrm{~mL})$ and prolonged hospitalization $[7,8]$.

We discussed the use of numerous approaches throughout the treatment of our patient. We wanted to take advantage of the fact that the anterior approach could be used to simultaneously treat C6-7 and T1-2 regions. Posterior approaches were ruled out due to the anterior pathologies of our patient. The multiple oblique corpectomy (MOC) approach is an interesting technique since it provides wide anterior decompression of the spinal canal and limited bone resection, which preserves spinal
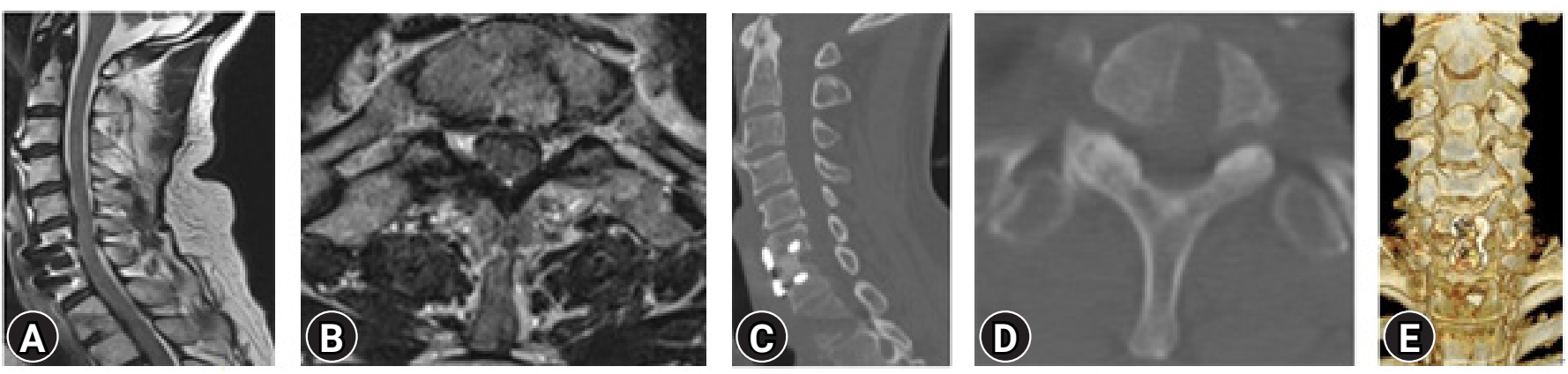

Figure 3. (A). Postoperative T2 sagittal magnetic resonance image (MRI) revealing the disappearance of herniated discs at C6-7 and T12, with no signs of cord compression. At the T1 body, the keyhole trajectory is shown. (B) A postoperative T2 axial MRI showing the T1 keyhole trajectory and disappearance of the T1-2 herniated disc, without signs of cord compression. (C) A sagittal computed tomography (CT) image showing adequate C6-7 anterior cervical decompression and fusion and a T1 keyhole trajectory. (D) Axial CT of the T1 keyhole trajectory is shown. (E) A three-dimensional CT of the anterior plate at C6-7 and the keyhole in the antero-superior part of the T1 vertebral body is shown. 
motion without causing instability [9]. The oblique approach would have been an ideal option for approaching C6-7 and T12; however, it was not selected because of its use may have required us to sacrifice the C7-T1 vertebral body or disc. Another interesting approach is keyhole transuncal foraminotomy. The technique involves direct drilling of the base of the uncinated process toward the intervertebral foramen, without destroying disc tissue. The technique also allows the functional anatomy of the uncovertebral joint to be retained post-surgery, since the joint is allowed to remain largely intact [10]. When selecting an ideal surgical method, advantages of MOC and keyhole transuncal foraminotomy were combined. A single Smith-Robinson incision of $5 \mathrm{~cm}$ was sufficient for ACDF realization at the C6-7 level, and T1-2 discectomy was accomplished using the oblique keyhole transcorporeal approach. With its oblique and caudal trajectory, there was no need to expose the T1-2 body nor was there a need for sternotomy, manubriectomy or claviculectomy. The oblique direction of keyhole entry (lateral to central and cephalad to caudal), the transition from lordosis to kyphosis at the C7-T1 level, and adequate positioning of the surgical bed made the perpendicular positioning of the drill possible, providing the surgeon with sufficient control and anatomic cues needed to reach the target point. Also, the keyhole approach does not cause instability because drilling of only a small portion of the vertebral body and endplate are required when performing a discectomy.

Detailed preoperative planning is needed to treat cases similar to the one described in this report. Decisions made were supported by fluoroscopic images obtained intraoperatively, which allowed the surgeon to continually check the disc level and the trajectory of the tunnel. This prevented unnecessary vertebral body resection while the surgeon attempted to reach the herniated disc at T1-2. Since the T1-2 level is hidden by the shoulder and other structures, c-arm oblique views to confirm the exact location of the drill when starting the tunnel were needed. An advanced intraoperative imaging system such as an $\mathrm{O}$-arm or navigation system would have made the procedure easier. However, these types of systems are not commonly available. Also, not all of T1-2 disc herniations can be treated by transcorporeal approach. Neck length varies on each patient and therefore preoperative planning is very important. We think that a good clinical diagnosis, meticulous preoperative planning, basic preoperative images such as MRI and CT, intraoperative fluoroscopic assistance, and an experienced surgeon are sufficient for overcoming most problems associated with the procedure. With adequate preoperative planning and ideal anatomic characteristics (patients with a long neck, those within whom it is possible to visualize the upper portion of the T2 body) we think that the oblique keyhole transcorporeal approach may be used to treat different disk levels, even at lower levels including T2-T3.

\section{CONCLUSION}

We report an extremely rare case of C6-7 cervical, and T1-2 thoracic tandem disc herniation successfully treated with C6-7 ACDF and T1-2 transcorporeal oblique keyhole discectomy using single-incision, single-stage surgery. With extensive preoperative planning, transcorporeal oblique keyhole discectomy is an attractive alternative to the anterior approach to treat T1-2 disc herniation. The procedure can be performed without sternotomy or manubriectomy, which are considered principal limitations of the anterior approach. We are concerned that all of T1-2 disc herniations can be treated by transcorporeal approach. There are anatomic variance within each patient such as neck length that may limit the transcorporeal approach. However, to our knowledge, this is the first case of cervico-thoracic tandem disc herniation treated with single-incision, single-stage surgery via an anterior approach with great result which might help other colleagues facing similar scenarios.

\section{CONFLICT OF INTEREST}

No potential conflict of interest relevant to this article.

\section{REFERENCES}

1. Mazas S, Benzakour A, Castelain JE, Damade C, Ghailane S, Gille O. Cervical disc herniation: which surgery? Int Orthop 2019;43:761-766.

2. Choi KC, Ahn Y, Lee CD, Lee SH. Combined anterior approach with transcorporeal herniotomy for a huge migrated cervical disc herniation. Korean J Spine 2011;8:292-294.

3. Gokcen HB, Erdogan S, Gumussuyu G, Ozturk S, Ozturk C. A rare case of T1-2 thoracic disc herniation mimicking cervical radiculopathy. Int J Spine Surg 2017;11:30.

4. Hurley ET, Maye AB, Timlin M, Lyons FG. Anterior versus posterior thoracic discectomy: a systematic review. Spine (Phila Pa 1976) 2017;42:E1437-E1445.

5. Oltulu I, Cil H, Berven S, Chou D, Clark A, Ulu MO, et al. Surgical management of thoracic disc herniation: anterior vs posterior approach. Turk Neurosurg 2019;29:584-593.

6. Son ES, Lee SH, Park SY, Kim KT, Kang CH, Cho SW. Surgical treatment of T1-2 disc herniation with $\mathrm{T} 1$ radiculopathy: 
a case report with review of the literature. Asian Spine J 2012;6:199-202.

7. Baranowska J, Baranowska A, Baranowski P, Rybarczyk M. Surgical treatment of thoracic disc herniation. Pol Merkur Lekarski 2020;49:267-270.

8. Choi BK, Han IH, Cho WH, Cha SH. Inferiorly migrated disc fragment at T1 body treated by $\mathrm{T} 1$ transcorporeal approach. J Korean Neurosurg Soc 2011;49:61-64.
9. Bruneau M, Cornelius JF, George B. Multilevel oblique corpectomies: surgical indications and technique. Neurosurgery 2007 61:106-112. discussion 112

10. Lee JY, Löhr M, Impekoven P, Koebke J, Ernestus RI, Ebel H, et al. Small keyhole transuncal foraminotomy for unilateral cervical radiculopathy. Acta Neurochir (Wien) 2006;148:951958. 